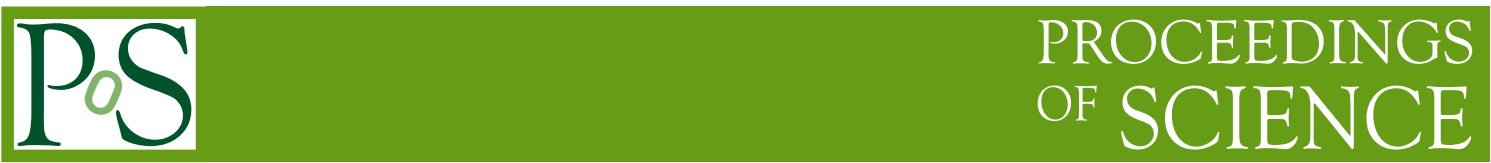

\title{
Dust observations with the new ALMA Band 1 receiver
}

\author{
Oscar Morata ${ }^{* a}$, James Di Francesco ${ }^{b}$, Ciska Kemper $^{a}$, and the ALMA Band 1 \\ Science Team \\ ( ${ }^{a}$ ) Academia Sinica Institute of Astronomy and Astrophysics (ASIAA), Taiwan \\ $\left.{ }^{b}\right)$ National Research Council-University of Victoria \\ E-mail: omorata@asiaa.sinica.edu.tw
}

The ALMA Band 1 project will expand the Atacama Large Millimeter/submillimeter Array (ALMA) access to frequencies between 35 and $52 \mathrm{GHz}$ for high angular resolution and sensitivity observations from the southern hemisphere. The main dust related science case for ALMA Band 1 is also an ALMA Level One Science Case: the study of the evolution of grains in protoplanetary disks. ALMA Band 1 will be able to resolve protoplanetary disks at the distance of the nearest star-forming regions and will allow us to follow the dust grain growth from mm-sized to $\mathrm{cm}$-sized pebbles in protoplanetary disks and hopefully show where and when dust coagulation occurs. Observations of debris disks will also be possible, although more challenging than those for protoplanetary disks. The high sensitivity and angular resolution of Band 1 will also allow us to study the spinning dust emission that it is related to the very small grain (VSG) population in the interstellar medium under conditions not possible to observe using mid-IR emission.

The Life Cycle of Dust in the Universe: Observations, Theory, and Laboratory Experiments - LCDU 2013, 18-22 November 2013

Taipei, Taiwan

* Speaker. 


\section{The ALMA Band 1 receiver}

The main goal of the ALMA Band 1 receiver is to provide access to frequencies $\sim 40 \mathrm{GHz}$ to the Atacama Large Millimeter/submillimeter Array (ALMA) at high angular resolution and sensitivity. A large set of compelling science cases proposed by the astronomical community for ALMA Band 1 are discussed in the Band 1 Science Case document [3]. The scope of these science cases is very wide, ranging from nearby stars to the re-ionization edge of the Universe. Two main ALMA Band 1 Science Cases are aligned with two ALMA Level One Science Goals: $i$ ) the study of the evolution of grains in protoplanetary disks through continuum observations down to $35 \mathrm{GHz}$ ( 9mm); and ii) detecting $\mathrm{CO}(3-2)$ line emission from galaxies during the epoch of re-ionization $(6<z<10)$. In fact, Band 1 will increase the volume of the observable Universe in CO lines by a factor of 8. ALMA Band 1 will also be a significantly faster imaging and surveying instrument than the Jansky VLA (JVLA); it will provide access to the southern sky; and it will make excellent science possible even in "poor" weather conditions.

The current planned frequency range that the Band 1 receiver will cover ranges from 35 to $52 \mathrm{GHz}$ (35-50 GHz within full ALMA specs and 50-52 GHz on a best effort basis). Compared with the previous frequency range specification for Band 1, this frequency range will provide a wider range of (slightly lower) redshifts in continuum and spectral line. It also adds the CS (1-0) line at $49 \mathrm{GHz}$, and some interesting molecules at 50-52 GHz (HDO, $\mathrm{C}_{3} \mathrm{H}_{2}$, etc). The continuum observations will have (slightly) improved angular resolution; and, finally, there will be a wider range of available frequencies, $\sim 17 \mathrm{GHz}$.

As mentioned above, the ALMA Band 1 covers part of the frequency range already observable, from the northern hemisphere, with the JVLA in the $\mathrm{K}_{a}$ and Q bands. The ALMA Band 1 Science Case document provides a comprehensive comparison of the properties and expected performance of ALMA and the JVLA over 40-50 GHz. In summary, ALMA Band 1 will have similar sensitivity at lower frequencies, but a slightly higher sensitivity (up to a factor of $\sim 2$ ) at higher frequencies; ALMA will have better mosaicking and imaging (better fidelity) capabilities than the JVLA, due to the larger primary beams and number of instantaneous baselines; and ALMA will be able to recover more extended emission (plus the possibility of using the Atacama Compact Array, ACA, and total power antennas to recover the shorter baselines).

\section{Dust related science cases for ALMA Band 1}

The three main science cases that have been proposed for ALMA Band 1 that directly involve dust observations are the study of dust around protoplanetary disks, the observation of debris disks and the detection of Very Small Grains (VSGs). We show below a shorter summary for each case. For a deeper discussion of each case, please refer to the ALMA Band 1 Science Case[3].

\subsection{Dust around protoplanetary disks}

Planet formation takes place in disks of dust and gas surrounding young stars. It is in protoplanetary disks where dust grains must agglomerate from sub-micron sizes to larger pebbles, rocks and planetesimals, if planets are to be formed, on a timescale of tens of Myr for terrestrial planets, although the process that leads to the formation of giant planet cores is still uncertain (from less 


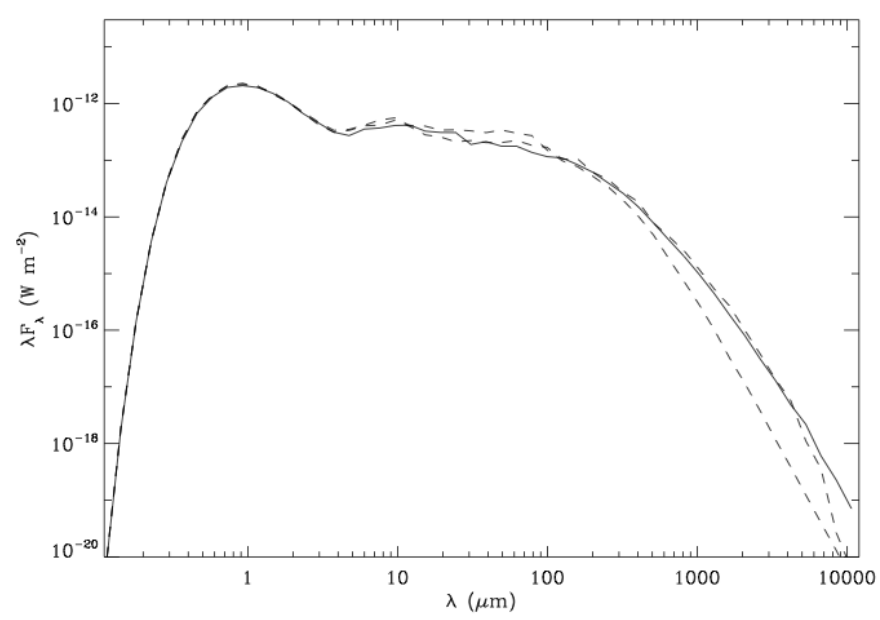

Figure 1: Spectral energy distribution plot showing the differences between three disk models having different maximum grain sizes: $a_{\max }=1 \mathrm{~cm}$ (solid), which keeps declining with roughly constant slope all the way to $1 \mathrm{~cm} ; \mathrm{a}_{\max }=10 \mu \mathrm{m}$ (bottom dashed line) and $1 \mathrm{~mm}$ (top dashed line). The fluxes are very much the same for $\mathrm{a}_{\max }=1 \mathrm{~mm}$ or $1 \mathrm{~cm}$, except precisely towards ALMA's Band 1, where there is at least an order of magnitude difference in power. Observations at the ALMA Band 1 regime are crucial for determining whether grain-growth to cm-sizes is indeed occurring.

than 1 Myr in dynamical instability models[1] to a few Myr in core accretion models [7]). The identification of where and when dust coagulation occurs is critical to constrain current models of planetary formation. The growth from sub-micron to micron-sized particles can be traced with IR spectroscopy and imaging polarimetry. But, ALMA Band 1 will be the only facility to be able to follow the evolution of dust grain growth from mm-sized to $\mathrm{cm}$-sized pebbles in protoplanetary disks.

Figure 2.1 shows the importance of Band 1 observations to determine whether grain-growth to $\mathrm{cm}$-sizes is occurring. Figure 2.1 shows the the spectral energy distributions (SEDs) of three different circumstellar disk models with different maximum grain sizes. The flux density at $1 \mathrm{~cm}$ of the model with the largest maximum grain size $(1 \mathrm{~cm})$ is $\sim 50 \mu \mathrm{Jy}$, comparable to the $1 \sigma$ sensitivity of Band 1 with 1 minute integration. Thus, ALMA Band 1 has a crucial role in the characterization of the coagulation processes of dust grains to centimeter sizes in protoplanetary disks. The possibility of comparing the Band 1 observations with those of other ALMA bands, under similar spatial frequency coverage conditions, gives an additional edge to Band 1 with respect to the JVLA.

ALMA Band 1 will also be able to resolve protoplanetary disks at the distance of the closest star-forming regions $(50-150 \mathrm{pc})$ using its longest baselines $\left(\sim 0.14^{\prime \prime}\right.$ at $\left.\sim 40 \mathrm{GHz}\right)$. The resolved images will provide a very accurate determination of the dust mass of the disk and will reveal, comparing with millimeter and submillimeter images, where the disk coagulation is happening.

\subsection{Debris disks}

Pebble-sized bodies are produced around main sequence stars by destructive collisional cascades from larger planetesimals through $\mathrm{cm}-, \mathrm{mm}-$, and $\mu \mathrm{m}$-sized particles that keep replenishing 
the debris population $[9,5]$. Similarly to the case of protoplanetary disks, the larger the grains that are present in the system, the longer the wavelength at which we must observed to detect their emission. ALMA Band 1 will be able to detect the larger particles that dominate the emission of debris disks, and may help detect edges or gaps in the disks, which could point to the existence of planets. The longer resonant lifetimes of the larger particles that dominate the emission of debris disks will help Band 1 obtain images with higher contrast features than other ALMA bands. Detection of debris disks in Band 1 will be challenging due to their relatively low surface brightness and large spatial distributions (hundreds of AU in radius). ALMA's large field-of-view, its mosaicking capabilities and the use of the ACA will provide higher sensitivity to the low surface brightness emission of these objects.

\subsection{Very small grains and spinning dust}

The emission of bright centimeter radio emission from several Galactic objects, most notably dark clouds $[6,2,8]$ has been explained by electric dipole radiation from rapidly rotating (spinning) very small dust grains (VSGs)[4]. This emission has also been seen as a large-scale foreground in CMB maps, spatially correlated with thermal dust emission and peaking at $\sim 40 \mathrm{GHz}$ (frequencies ideally covered by Band 1).

Directly measuring the VSG abundance and solid state physics will be very exciting, because VSGs are thought to play a central role in the chemical and thermal balance of the ISM. Moreover, the population of VSGs may have implications for disk evolution and sedimentation. The high angular resolution and excellent sensitivity of ALMA Band 1 are ideal for differentiating the distinct environments where the VSG population resides and will be crucial for probing their presence in star formation and circumstellar regions.

\section{References}

[1] A. Boss, Evolution of the Solar Nebula. VII. Formation and Survival of Protoplanets Formed by Disk Instability, ApJ, 629, 535 (2005)

[2] S. Casassus, C. Dickinson, K. Cleary, et al., Centimetre-wave continuum radiation from the ÏA Ophiuchi molecular cloud, MNRAS, 391, 1075 (2008)

[3] J. Di Francesco, D. Johnstone, B. C. Matthews, et al., The Science Cases for Building a Band 1 Receiver Suite for ALMA [arXiv: 1310.1604 ]

[4] B. T. Draine, A. Lazarian, Diffuse Galactic Emission from Spinning Dust Grains, ApJ, 494, 19 (1998)

[5] C. P. Dullemond, C. Dominik, Dust coagulation in protoplanetary disks: A rapid depletion of small grains, $A \& A, \mathbf{4 3 4}, 971$ (205)

[6] D. P. Finkbeiner, D. J. Schlegel, C. Frank, C. Heiles, Tentative Detection of Electric Dipole Emission from Rapidly Rotating Dust Grains, ApJ, 566, 898 (2002)

[7] J. Pollack, O. Hubickyj, P. Bodenheimer, et al., Formation of the Giant Planets by Concurrent Accretion of Solids and Gas, Icarus, 124, 62 (1996)

[8] AMI Consortium, A. M. M. Scaife, N. Hurley-Walker, et al., AMI observations of Lynds dark nebulae: further evidence for anomalous cm-wave emission, MNRAS, 400, 1394 (2009)

[9] M. C. Wyatt, Evolution of Debris Disks, ARA\&A, 46, 339 (2008) 